This document is published in:

Robles, G.; Martinez-Tarifa, J.M.; Rojas-Moreno, M.V.; Albarracin, R.; Ardila-Rey, J.; , "Antenna selection and frequency response study for UHF detection of partial discharges," Instrumentation and Measurement Technology Conference (I2MTC), 2012 IEEE International , vol., no., pp.1496-1499, 13-16 May 2012. DOI: 10.1109/I2MTC.2012.6229440

(C) 2012 IEEE. Personal use of this material is permitted. Permission from IEEE must be obtained for all other uses, in any current or future media, including reprinting/republishing this material for advertising or promotional purposes, creating new collective works, for resale or redistribution to servers or lists, or reuse of any copyrighted component of this work in other works. 


\title{
Antenna selection and frequency response study for UHF detection of partial discharges
}

\author{
Robles, G.; Martínez-Tarifa, J.M.; Rojas-Moreno, M.V.; Albarracín, R.; Ardila-Rey, J. \\ Department of Electrical Engineering \\ Universidad Carlos III de Madrid \\ Leganés, Spain \\ grobles@ing.uc3m.es
}

\begin{abstract}
Partial Discharge (PD) detection is a widely extended technique for electrical insulation diagnosis. Classical PD detection by means of phase resolved patterns require electrical connections to the power equipment and is sensitive to many noise sources. Ultra High Frequency (UHF) detection techniques are being recently proposed to overcome these problems, and to detect partial discharges on-line. In this paper, four antennas will be tested in order to compare their response to this physical phenomenon.
\end{abstract}

Keywords- partial discharges; UHF detection; antennas response.

\section{INTRODUCTION}

Partial Discharge (PD) is a clear ageing agent in electrical insulation of power systems. Power cables, transformers and generators withstand PD even at rated voltages due to mixed ageing agents arising from thermal, mechanical, electrical and environmental stresses [1]. On the other hand, these microscopic ionizations lead to small signal current pulses that can be detected in electrical equipment. Thus, PD is also a symptom of high voltage electrical apparatus ageing.

Normalized PDs measurements are made using resistivecapacitive dividers [2]. These classical methods use PhaseResolved PD (PRPD) patterns to identify certain PD sources (corona, internal and surface PD). However, the measurement procedure is always done in industrial environments, where low Signal-to-Noise Ratio (SNR) signals are obtained or several microscopic sites are usually discharging simultaneously. This is the reason to add new measurement techniques where PD pulse waveform analysis is a fruitful methodology for noise rejection and PD source separation [3]. In this case, Very High Frequency (VHF) detectors, such as High Frequency Current Transformers (HFCT), are usually selected.

However, these methods have drawbacks: power equipment disconnection is sometimes necessary to adjust the measurement and detection of discharges and PD geometric location is not possible with these systems. In order to solve this, acoustic and UHF PD detection techniques are recently being applied to these systems, since these are non-contact measurements that can help in on-line PD monitoring and location [4]-[7]. Unfortunately, acoustic detection is restricted to oil-paper insulation systems and inner PD sites can be hardly detected because acoustic waves only propagate through oil.

Despite the fact that UHF detection seems to have clear advantages over other techniques, it is not fully understood the relationship between conventional HF or VHF signals and UHF signals [8]. Moreover, UHF signals depend on the selected antenna bandwidth and gain. Hence, the selection of the proper antenna for PD detection and location in high voltage assets is currently a clear technical challenge.

In this paper, a study about UHF signals from controlled insulating test objects withstanding PD is proposed. Four different antennas: zig-zag, monopoles with two lengths and log-periodic, will be used to measure PD pulses and their responses will be compared in the frequency domain. Discussion will be made taking into account technical and economic characteristics for each device.

\section{MEASURING SETUP}

The measuring setup consists of two different parts: partial discharge generation and sensor deployment around the test object.

\section{A. Partial Discharge generation}

A constant and predictable partial discharges activity is necessary to ensure the repeatability of results. To achieve this, a controlled experiment is carried out in the laboratory with a test object consisting of a vessel filled with transformer oil and two electrodes separated by 5 sheets of transformer insulating paper, see Fig. 1.

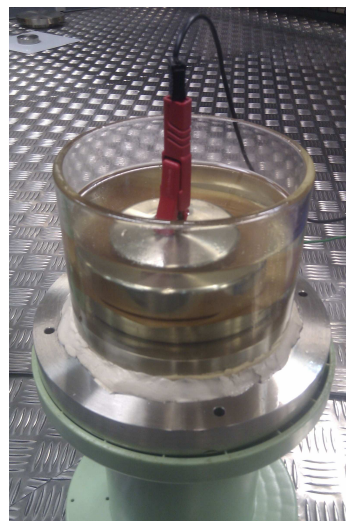

Figure 1. Test object

This research has been supported by the Madrid Regional Government and Universidad Carlos III de Madrid under Contract No. CCG10UC3M/DPI-4627. Tests have been carried out in the High Voltage Research and Tests Laboratory at Universidad Carlos III de Madrid (LINEALT). 


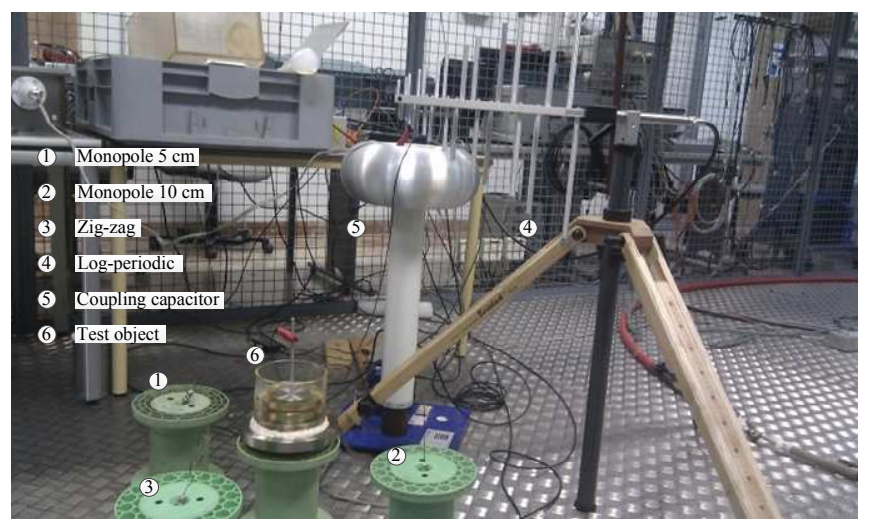

Figure 2. Measuring setup with four antennas. The test object and the coupling capacitor are also visible

An electrode is connected to a high voltage source and the other to ground. According to Standard IEC 60270, a coupling capacitor is connected in parallel to the test object to provide a path to ground for high frequency current pulses created by partial discharges in the test object, see Fig. 2. Pulses are measured in VHF with a HFCT with a bandwidth up to 40 $\mathrm{MHz}$ connected to a commercial PDs detector ( $P D$-Check from TechImp Systems S.r.l.) capable to identify PRPD patterns. This test setup in VHF is used to confirm that the detected UHF pulses are a consequence of PDs activity.

It has been found, that using new and dry transformer paper sheets, the partial discharges activity (internal PDs in microscopic air voids between papers) starts around $2 \mathrm{kV}$ and is stable during at least three hours which is enough to acquire proper signals in VHF with the HFCT and in UHF with the antennas. Hence, the high voltage source is slowly set slightly above the inception voltage and the measuring campaign starts. Pulses were acquired at $3600 \mathrm{~V}$.

\section{B. Antennas deployment}

Four antennas are used in the experiments with different frequency ranges: UHALP 91088A log-periodic from 250 to $2400 \mathrm{MHz}$, two monopoles, 5 and $10 \mathrm{~cm}$ long, which ensures a wide frequency range [7], and a zig-zag antenna, see Fig. 2 and Fig. 3. The antennas are deployed around the test object and their outputs connected to an oscilloscope. The distances between the test object and the antennas are not critical parameters at this stage because the experiment is focused on studying the frequency response of the signals and not the pulses in the time domain; nevertheless, distances were of a similar range, as can be seen in Fig. 2. Since PD source location is not the focus of this paper, the lengths of the coaxial cables are random too.

UHF acquisitions were made in a Tektronix DPO7254 8bit, $40 \mathrm{GS} / \mathrm{s}$, 4 channel oscilloscope, where the response of each antenna to PD pulses was registered. PDs activity is a stochastic phenomenon that depends on several factors such as applied voltage level, insulation ageing status, environmental conditions, etc. Despite the fact that, during the experiments in laboratory, most factors were controlled to assure uniformity in

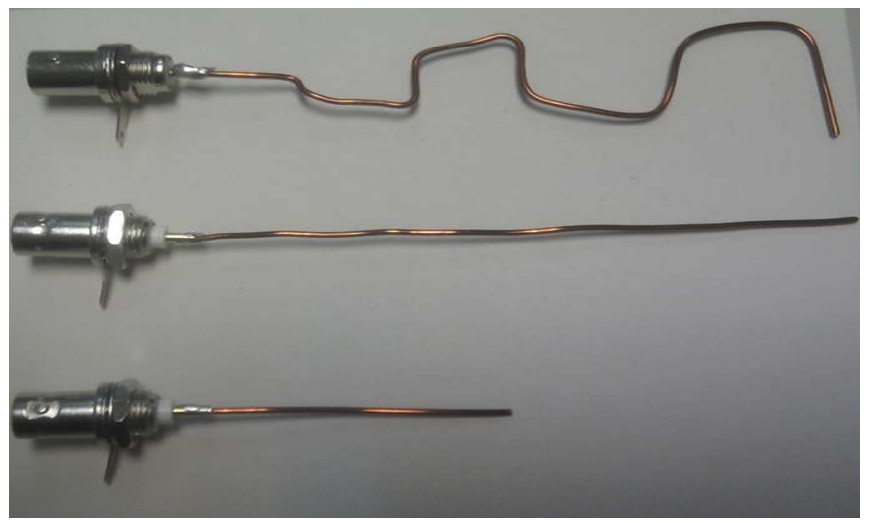

Figure 3. Zig-zag, $10 \mathrm{~cm}$ monopole and $5 \mathrm{~cm}$ antennas

the measurements, series of 500 pulses were recorded and processed to guarantee that the results were statistically reliable.

\section{Signal ACQUiSITION AND PROCESSING}

Prior to starting the acquisition of partial discharge pulses, the background noise is characterized to be compared with the FFT in the presence of pulses. This is done by measuring the
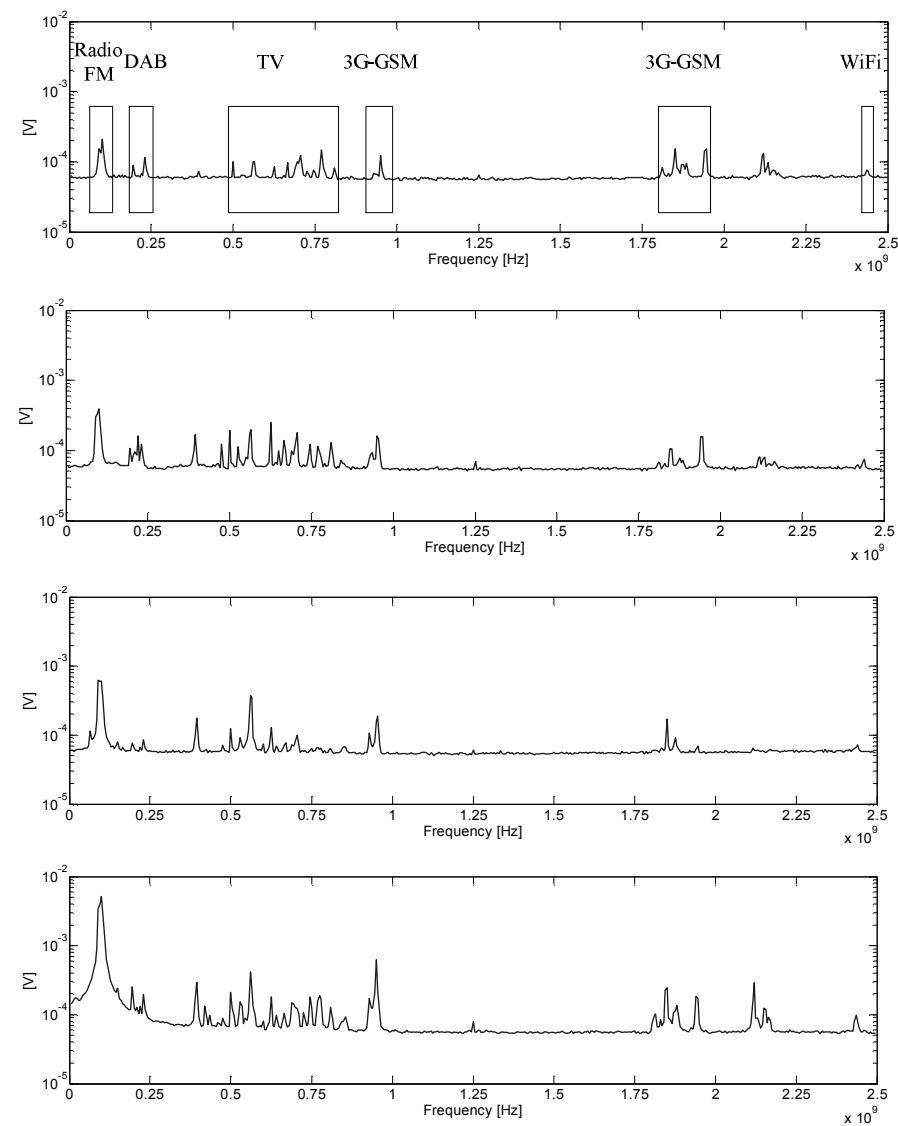

Figure 4. FFT voltage amplitude. Top: $5 \mathrm{~cm}$ monopole antenna; Second one: $10 \mathrm{~cm}$ monopole antenna. Third one: zig-zag antenna.; Bottom: log-periodic antenna 

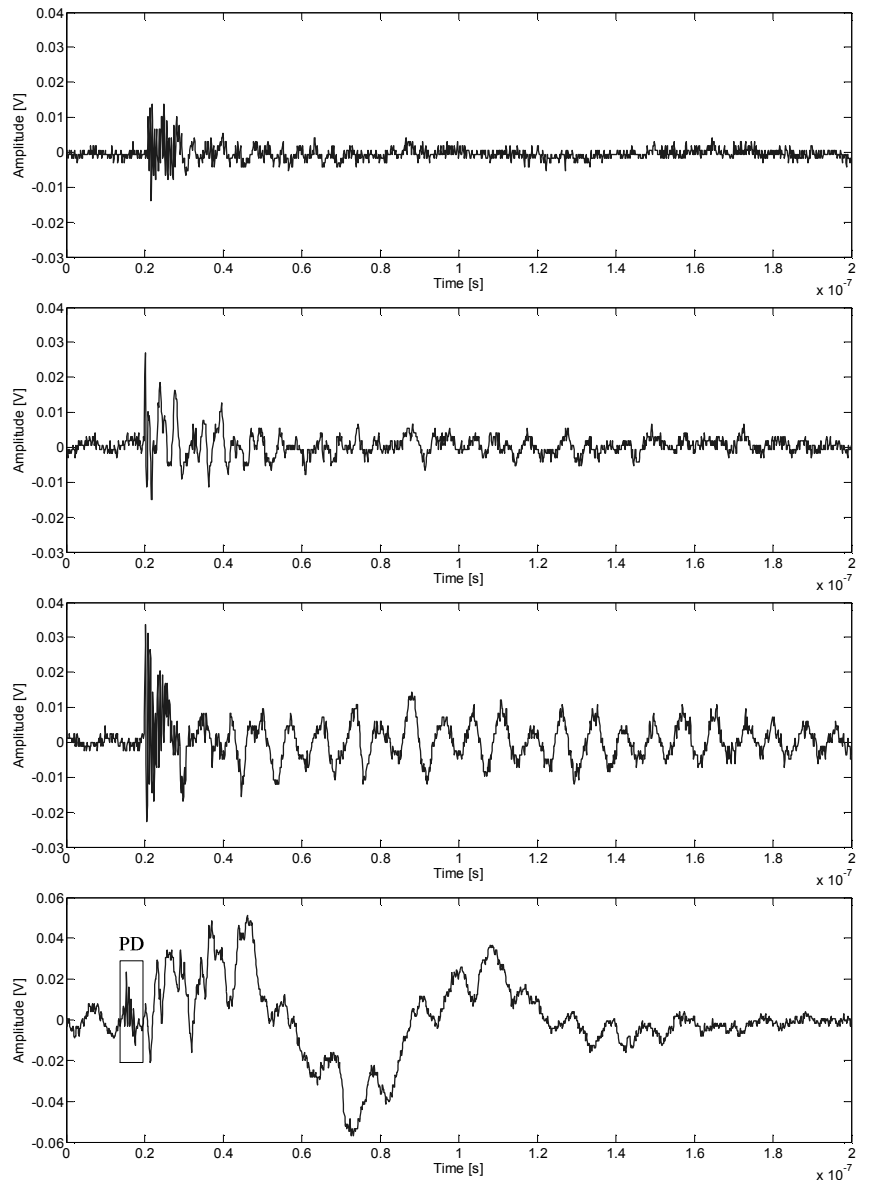

Figure 5. Voltage amplitud versus time for PD at $3600 \mathrm{~V}$. Top: $5 \mathrm{~cm}$ monopole antenna; Second one: $10 \mathrm{~cm}$ monopole antenna. Third one: zig-zag antenna.; Bottom: log-periodic antenna

amplitude in absence of applied high voltages and shown in Fig. 4, where the FM radio, Digital Audio Broadcasting (DAB), TV broadcast, GSM and WiFi signals are clearly visible. The vertical axis for the four antennas is set to a logarithmic scale in volts. The noise floor is around $6 \cdot 10^{-5} \mathrm{~V}$, $5.8 \cdot 10^{-5} \mathrm{~V}, 6 \cdot 10^{-5} \mathrm{~V}$ and $7 \cdot 10^{-5} \mathrm{~V}$ for the $5 \mathrm{~cm}$ monopole, 10 $\mathrm{cm}$ monopole, zig-zag and log-periodic antennas, respectively. Using the FM band to compare the response of the antennas to external radiation, it can be observed that the peaks are located in $2 \cdot 10^{-4} \mathrm{~V}, 4 \cdot 10^{-4} \mathrm{~V}, 6 \cdot 10^{-4} \mathrm{~V}$ and $5 \cdot 10^{-3} \mathrm{~V}$ for the $5 \mathrm{~cm}$ monopole, $10 \mathrm{~cm}$ monopole, zig-zag and log-periodic antennas, respectively. This means that, considering wide band behaviour, the log-periodic antenna has better sensitivity than the others. The horizontal axis is the frequency in $\mathrm{Hz}$ with a scale of $250 \mathrm{MHz} / \mathrm{div}$, from 0 to $2.5 \mathrm{GHz}$. This plot is done by averaging the FFT of 500 time signals acquired with the oscilloscope.

Once this noise is characterized, the voltage is raised up to the inception voltage and the pulses are synchronized with the trigger set to channel one in the oscilloscope where the $10 \mathrm{~cm}$ monopole is connected. An example of the time domain signals acquired is shown in Fig. 5 for pulses at $3600 \mathrm{~V}$. The sampling frequency is $10 \mathrm{GS} / \mathrm{s}$, the acquisition time is $200 \mathrm{~ns}$ and the voltage peaks for the three signals are below $60 \mathrm{mV}$. Notice
TABLE I. Cumulative Power by Bands

\begin{tabular}{|l|c|c|c|}
\hline \multirow{2}{*}{ Antenna type } & \multicolumn{2}{|c|}{$\begin{array}{c}\text { Cumulative Power by Bands } \\
\left(\mathbf{V}^{2}\right)\end{array}$} & \\
\cline { 2 - 4 } & $\boldsymbol{0} \boldsymbol{~ V . 6 ~ k V}$ & Ratio \\
\hline $\begin{array}{l}5 \mathrm{~cm} \text { monopole } \\
0-600 \mathrm{MHz}\end{array}$ & $6.53 \cdot 10^{-7}$ & $1.98 \cdot 10^{-6}$ & 3.0 \\
\hline $\begin{array}{l}5 \mathrm{~cm} \text { monopole } \\
1300-1900 \mathrm{MHz}\end{array}$ & $2.51 \cdot 10^{-7}$ & $3.661 \cdot 10^{-6}$ & 14.6 \\
\hline $\begin{array}{l}10 \mathrm{~cm} \text { monopole } \\
0-600 \mathrm{MHz}\end{array}$ & $3.62 \cdot 10^{-7}$ & $1.034 \cdot 10^{-5}$ & 28.6 \\
\hline $\begin{array}{l}10 \mathrm{~cm} \text { Monopole } \\
1300-1900 \mathrm{MHz}\end{array}$ & $3.20 \cdot 10^{-7}$ & $1.82 \cdot 10^{-6}$ & 5.7 \\
\hline $\begin{array}{l}\text { Zig-zag } \\
0-600 \mathrm{MHz}\end{array}$ & $1.30 \cdot 10^{-6}$ & $2.96 \cdot 10^{-5}$ & 22.8 \\
\hline $\begin{array}{l}\text { Zig-zag } \\
1300-1900 \mathrm{MHz}\end{array}$ & $2.57 \cdot 10^{-7}$ & $1.90 \cdot 10^{-6}$ & 7.4 \\
\hline $\begin{array}{l}\text { Log-periodic } \\
0-600 \mathrm{MHz}\end{array}$ & $4.22 \cdot 10^{-5}$ & $3.13 \cdot 10^{-4}$ & 7.4 \\
\hline $\begin{array}{l}\text { Log-periodic } \\
1300-1900 \mathrm{MHz}\end{array}$ & $3.86 \cdot 10^{-7}$ & $1.87 \cdot 10^{-6}$ & 4.8 \\
\hline
\end{tabular}

that the signal for the log-periodic antenna is detected before the signals of the monopoles and zig-zag antennas. This is due to the fact that, even when the monopoles and zig-zag are closer to the test object, they have longer coaxial cables, $(3 \mathrm{~m}$ long for log-periodic antenna and $5 \mathrm{~m}$ long for the others), connected to the oscilloscope.

The same FFT analysis is done for signals with pulses from partial discharges and the result is shown in Fig. 6. It is a remarkable fact that the amplitudes of the spectra in the low frequency range of the FFT, up to $600 \mathrm{MHz}$, are noticeably larger. However, the importance of these results lays on the FFT content that appears in the range from 1300 to $1900 \mathrm{MHz}$ and that is directly related to the UHF emission of the partial discharge pulse, compare Fig. 6 and Fig. 4. These peaks are captured by the four antennas being the $5 \mathrm{~cm}$ monopole antenna the best one to visualize them. Moreover, it is also remarkable the performance of the $10 \mathrm{~cm}$ monopole considering its simplicity and inexpensive manufacture compared to the log-periodic antenna.

Table I. summarizes the behavior of the antennas showing the cumulative power by bands, in order to make a comparison between the frequency response of the antennas, in two ranges of frequency: from 0 to $600 \mathrm{MHz}$ and from 1300 to $1900 \mathrm{MHz}$. The column on the right represents the ratio between the power content in squared volts in those ranges with $\mathrm{PD}$ and without PD. It can be clearly seen that the spectral power of the PD pulses detected with the antennas is notably larger than in the case of absence of PD, specially, in the lower frequency band of study, from 0 to $600 \mathrm{MHz}$. The best frequency response in this band is for the $10 \mathrm{~cm}$ monopole, and the zig-zag antennas, reaching a ratio of 28.6 , and 22.8 respectively. In the higher frequency band, from 1300 to $1900 \mathrm{MHz}$, the cumulative power increase due to PDs activity is not so high but is more relevant because there is energy only when PD occurs otherwise, this band is flat. In this band, the best behavior is found for the $5 \mathrm{~cm}$ monopole with a remarkable ratio of 14.6. The $10 \mathrm{~cm}$ monopole and the zig-zag antennas only reach ratios of 5.7 and 7.4, respectively. The differences between 10 $\mathrm{cm}$ and $5 \mathrm{~cm}$ monopoles arise from their different sensitivity to 

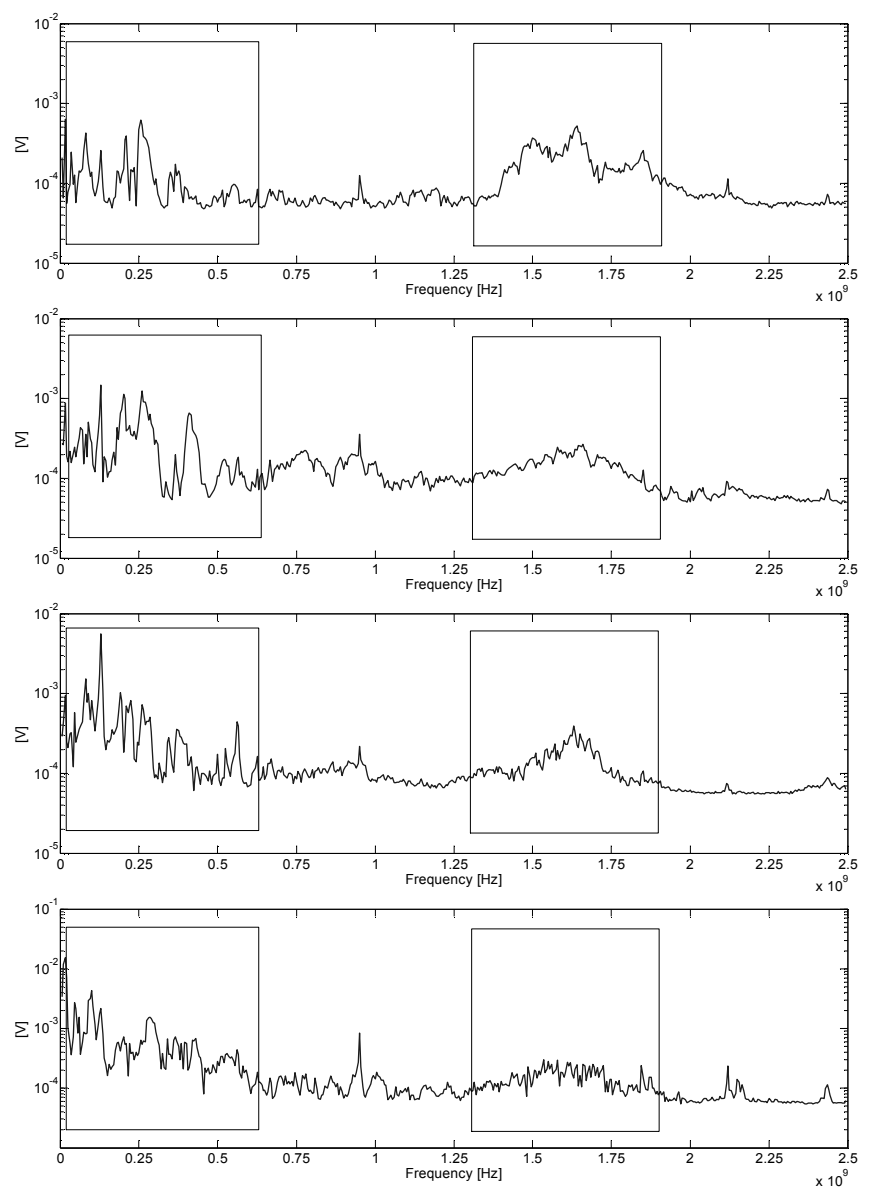

Figure 6. FFT voltage amplitude during the presence of PD at $3600 \mathrm{~V}$. Top: $5 \mathrm{~cm}$ monopole antenna; Second one: $10 \mathrm{~cm}$ monopole antenna. Third one: zig-zag antenna.; Bottom: log-periodic antenna

electromagnetic radiation for different wavelengths, so the 5 $\mathrm{cm}$ monopole must have a better response at higher frequencies.

The poor performance of the log-periodic antenna can be seen in Fig. 5 where the direct electromagnetic wave of the PD had to be highlighted putting it into a box. These types of antennas capture PD and signals without diagnosis interest in a broadband of frequencies, and PD, generally with low emitting powers, are hidden by the rest of signals.

For all these reasons, if measurements are taken in noisy electromagnetic environments with high Radio Frequency (RF) spectral amplitudes, the $5 \mathrm{~cm}$ monopole would be the best option because it would detect PDs in the $1300-1900 \mathrm{MHz}$ band with good sensitivity.
This study opens a research trend to characterize the behavior of monopole antennas with different lengths and shapes. The frequency response for these inexpensive monopoles shows a broadband behavior appropriate for UHF emissions from insulation systems. Their significant components above $1.3 \mathrm{GHz}$ are an interesting characteristic for PDs location, where the geometric sensitivity is determined by the bandwidth of the antennas. Thus, several inexpensive monopoles can be used for PDs sites location in power systems.

\section{CONCLUSION}

The $10 \mathrm{~cm}$ monopole and zig-zag antennas show an overall better performance in the two frequency bands of study after comparing the behaviour of the four antennas. However, the 5 $\mathrm{cm}$ monopole antenna is the best detecting PDs emitting in UHF from 1300 to $1900 \mathrm{MHz}$ band. Monopoles and zig-zag antennas are strong candidates for further studies because these antennas are inexpensive, simpler, smaller, easier to manufacture and can be tuned to a frequency band of interest by changing their lengths. The paper also gives practical indications about PDs detection with different antennas.

\section{REFERENCES}

[1] G. Stone, E.A. Boutler, I. Culbert, H. Dhirani, "Electrical insulation for rotating machines: design, evaluation, aging, testing and repair". New Jersey; IEEE Press Series on Power Engineering, Wiley Interscience; 2004;p. 295-307.

[2] IEC Document No 60270, "High voltage test techniques-Partial discharge measurements", 2000.

[3] A. Cavallini, G.C. Montanari, A. Contin, F. Pulletti, "A new approach to the diagnosis of solid insulation systems based on PD signal inference," Electrical Insulation Magazine, IEEE , vol.19, pp.23-30, March-April 2003.

[4] J. Ramírez-Niño, A. Pascacio, “Acoustic measuring of partial discharges in power transformers," Measurement Science and Technology, 2009, vol. 20, 115108, November 2009.

[5] S. Markalous, S. Tenbohlen, K. Feser, "Detection and location of partial discharges in power transformers using acoustic and electromagnetic signals," Dielectrics and Electrical Insulation, IEEE Transactions on , vol.15, pp.1576-1583, December 2008.

[6] P.J. Moore, I. Portugues, I.A. Glover, "A nonintrusive partial discharge measurement system based on RF technology," Power Engineering Society General Meeting, 2003, IEEE, vol.2, pp. 4 vol. 2666, 13-17 July 2003.

[7] J. López-Roldán, T. Tang, M. Gaskin, "Optimisation of a sensor for onsite detection of partial discharges in power transformers by the UHF method," Dielectrics and Electrical Insulation, IEEE Transactions on , vol.15, pp.1634-1639, December 2008.

[8] A.J. Reid, M.D. Judd, R.A. Fouracre, B.G. Stewart, D.M. Hepburn, "Simultaneous measurement of partial discharges using IEC60270 and radio-frequency techniques," Dielectrics and Electrical Insulation, IEEE Transactions on, vol.18, pp.444-455, April 2011. 